\title{
USE OF LATERAL INTERCOSTAL PERFURATED FLAPS (LICAP) IN TUMORS OF THE UPPER EXTERNAL QUADRANT OF THE BREAST
}

María Laura Ramos Pérez¹, Carlos Ricardo Chagas¹, Fernando de Carvalho1, Martín Falla Jimenez²

${ }^{1}$ Pontifícia Universidade Católica - Rio de Janeiro (RJ), Brazil.

${ }^{2}$ Oncosalud - Auna, Peru.

Introduction: In the surgical treatment of breast cancer we have two great options: mastectomy and conservation surgery. The second one has been increasing over the last 30 years, since the safety of tumor resection with margins added to radiation therapy was demonstrated. Currently with the use of neoadjuvant therapy in advanced and early stages, the challenge for surgeons is not only to offer to preserve breast tissue, but also to ensure oncological safety with increasingly better aesthetic results. Most of the breast volume is concentrated in the upper external quadrant, therefore the removal of tumors in this location represents a significant loss in breast volume, causing asymmetries, affecting the contour of the breast and causing visible scars in most cases. On the other hand, in many patients with small breasts, the breast and tumor ratio is often unfavorable, leading to mastectomies. However, by using flaps for immediate reconstruction, this could change favorably for this group of patients. The use of techniques that involve lateral flaps, such as the lateral intercostal artery perforator flap (LICAP), could solve these problems, allowing for an adequate replacement of the resected volume. In this series of cases, considerable volumes have been removed, which have been replaced by LICAP, successfully achieving the treatment of tumors of the upper external quadrant of the breast. The use of this technique through a lateral incision guarantees, in addition to a wide resection in the breast, a practical and simple axillary approach, minimizing visible scars and optimizing the cosmetic result in these patients. Case report: Three cases were reported from two institutions (Casa da Saúde Nossa Senhora de Fátima, in Rio de Janeiro, and Oncosalud -Auna, in Peru). The patients were middle-aged women, all of them with tumors located in the upper external quadrant of the breast, one of the patients having received neoadjuvant therapy. In one of the cases, the breast volume was small, having an unfavorable breast / tumor ratio. By using LICAP, a favorable immediate reconstruction was achieved and a mastectomy was avoided. In one of the cases, an axillary dissection was performed and the other two underwent sentinel node biopsy; the three cases had a single lateral approach, by which a tumor resection of the breast and axillary surgery were performed. During the evolution of the patients, there were no complications, initiating treatment with radiotherapy in due time. The cosmetic result was optimal in terms of volume replacement, minimal scarring and breast symmetry. 